\title{
BMJ Open Chinese patent medicine Fei-Liu-Ping ointment as an adjunctive treatment for non-small cell lung cancer: protocol for a systematic review
}

Honggang Zheng, ${ }^{1}$ Shulin He, ${ }^{1,2}$ Rui Liu, ${ }^{1}$ Xinyao Xu, ${ }^{1,2}$ Tao Xu, ${ }^{1}$ Shuntai Chen, ${ }^{1,2}$ Qiujun Guo, ${ }^{1,2}$ Yebo Gao, ${ }^{1,2}$ Baojin Hua ${ }^{1}$

To cite: Zheng $\mathrm{H}$, He S, Liu R, et al. Chinese patent medicine Fei-Liu-Ping ointment as an adjunctive treatment for non-small cell lung cancer: protocol for a systematic review. BMJ Open 2017;7:e015045

doi:10.1136/bmjopen-2016015045

- Prepublication history for this paper is available online. To view these files please visit the journal online (http://dx.doi.org/10.1136/ bmjopen-2016-015045).

$\mathrm{HZ}$ and $\mathrm{SH}$ are co-first authors.

Received 8 November 2016 Accepted 19 December 2016

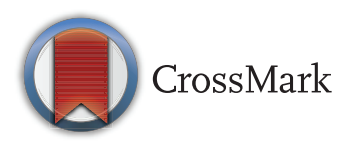

${ }^{1}$ Department of Oncology, Guang'anmen Hospital, China Academy of Chinese Medical Sciences, Beijing, China ${ }^{2}$ Beijing University of Chinese Medicine, Beijing, China

Correspondence to Professor Honggang Zheng; honggangzheng@126.com

\section{ABSTRACT \\ Introduction: Fei-Liu-Ping ointment has been widely applied as adjunctive drug in the treatment of non- small cell lung cancer (NSCLC). However, there has been no systematic review of research findings regarding the efficacy of this treatment. Here, we provide a protocol for assessing the effectiveness and safety of Fei-Liu-Ping ointment in the treatment of NSCLC.}

Methods and analysis: The electronic databases to be searched will include MEDLINE (PubMed), Cochrane Central Register of Controlled Trials (CENTRAL) in the Cochrane Library, Excerpt Medica Database (EMBASE), China National Knowledge Infrastructure (CNKI), China Scientific Journal Database (VIP), Wanfang Database and Chinese Biomedical Literature Database (CBM). Papers in English or Chinese published from inception to 2016 will be included without any restrictions. We will conduct a meta-analysis of randomised controlled trial if possible. The therapeutic effects according to the standard for treatment of solid tumours by the WHO and the quality of life as evaluated by Karnofsky score and weight will be applied as the primary outcomes. We will also evaluate the data synthesis and risk of bias using Review Manager 5.3 software.

Dissemination: The results of this review will offer implications for the use of Fei-Liu-Ping ointment as an adjunctive treatment for NSCLC. This knowledge will inform recommendations by surgeons and researchers who are interested in the treatment of NSCLC. The results of this systematic review will be disseminated through presentation at a conference and publication of the data in a peer-reviewed journal.

Trial registration number: PROSPERO CRD42016036911.

\section{DESCRIPTION OF THE CONDITION}

Lung cancer is a lethal cancer and remains a primary cause of cancer-related deaths in the USA among both genders, with non-small cell lung cancers (NSCLCs) accounting for

\section{Strengths and limitations of this study}

- We will objectively evaluate the efficacy and safety of Fei-Liu-Ping ointment for the treatment of non-small cell lung cancer (NSCLC) in this systematic review.

- Our review may provide evidence for researchers and be helpful for clinical practitioners in treating NSCLC.

- The systematic review will also have obvious limitations, especially the language bias. Medical studies prepared in Japanese and Korean will not be covered in this review.

$85 \%$ of lung cancer-related deaths. ${ }^{1}$ It is estimated that there will be 224390 new diagnoses of lung cancer and nearly 158080 deaths in 2016. ${ }^{2}$ The American Joint Committee on Cancer (AJCC) has developed a staging system to determine the development of cancer in the body. A list of three variables, that is, the initial tumour, spread to lymph nodes, and metastasis, is normally applied as the reference for staging. ${ }^{3}$ Generally, the staging system is beneficial for the doctor to plan treatment and predict prognosis. For example, staging in lung and bronchial cancer cases reflects different 5-year survival rates. $^{4}$

Lung cancer was first recorded in an ancient Chinese medical text 'The Yellow Emperor's Internal Classic' (Chinese name: 'Huang Di Nei Jing'). Based on the theory of traditional Chinese medicine (TCM), health qi deficiency was proposed to be responsible for lung cancer development. Qi is literally translated into gas or breath, which is the vital energy for human activity, similarly with immunity and vitality. TCM theory insists that the lung masters qi and breath. Abundant qi will enable the body to fight against diseases, while qi deficiency will cause pathogenic 
factors such as 'qi stagnation', 'blood stasis', 'phlegm', 'damp', and 'toxicity', which may contribute to the occurrence of lung cancer. Therefore, clinical practices focus on dispelling these pathogenic factors and strengthening qi at the same time.

\section{DESCRIPTION OF THE INTERVENTION}

Surgery is proposed as the best treatment for lung cancer. However, only about $20-25 \%$ of tumours are suitable for resection. ${ }^{5}$ Chemotherapy and radiotherapy were also thought to be the main treatments but are associated with serious side effects including toxicity to normal cells and tissues. ${ }^{6}$ Despite the discovery and application of targeted therapies, the overall survival remains poor, with an overall 5-year survival rate of $\sim 16.6 \%$. $^{7}$ Therefore, an ideal treatment regime for lung cancer is still required.

Traditional medicine (TM) is accepted worldwide as an alternative or adjunctive treatment to the conventional treatments. ${ }^{8}$ As reported, at least 30\% Americans see TM practitioners annually. Interestingly, TM has good efficacy in treating illnesses that are insensitive to conventional treatment. ${ }^{9}$ TCM is an important branch of TM, which follows the theoretical concepts of Yin-Yang and the five elements. Herbal medicine is widely applied in disease treatment based on TCM theory. There are several different ways to prepare herbal medicines, including Chinese patent medicine and herbal extract injection, doctor-prescribed decoction, classical decoctions described in ancient Chinese texts, and hospital patent formula. ${ }^{3}$ Fei-Liu-Ping (FLP) ointment was developed by Guang'anmen Hospital, China Academy of Chinese Medical Sciences and patented as one of the hospital patent formulas. Although FLP ointment is widely applied in China to treat NSCLC, there have been no systematic reviews evaluating its effectiveness and safety.

\section{HOW THE INTERVENTION MIGHT WORK}

FLP ointment is a Chinese patent medicine with a long history of application in China. FLP ointment consists of several medical herbs, with the main components being Astragalus membranaceus, American ginseng, Adenophora stricta, Radix Ophiopogonis, Oldenlandia diffusa, Bistort root, Patrinia, semen persicae and pseudoginseng. The action of As. membranaceus, American ginseng, Ad. stricta Miq, and Radix Ophiopogonis is to influence the immune system, suppress cancer cell migration, modulate various cancer signalling pathways, and interact with specific transcription molecules during protection against inflammation and cancers. ${ }^{10} 11$ The action of persicae and pseudoginseng is to activate blood, remove blood stasis, change the whole blood viscosity, reduce metastasis, and promote immune response. ${ }^{12}$ The other herbs, Oldenlandia diffusa, Bistort root and Patrinia, have the function of removing toxicity and exert antitumour effects. ${ }^{13}$ FLP ointment has been shown to have many anticancer activities, ${ }^{14}$ which include stimulating dendritic cells, ${ }^{15}$ inhibiting vascular endothelial growth factor (VEGF) signalling, ${ }^{16}$ modulating matrix metalloproteinase 9 (MMP9) and tissue inhibitor of metallopeptidase 1 (TIMP-1) expression, ${ }^{15}$ and regulating inflammation through interactions with the nuclear factor $\kappa \mathrm{B}(\mathrm{NF}-\kappa \mathrm{B})$ signalling pathway within the tumour microenvironment. ${ }^{16} 17$ According to TCM theory, FLP ointment can be used for the treatment of symptoms caused by a dual deficiency of qi, such as fatigue, excess sweating, etc. Patients always suffer from general weakness and fatigue after chemotherapy or radiotherapy. ${ }^{18}$ The symptoms are consistent to the syndrome of qi deficiency in TCM theory. In order to determine the safety and efficacy of FLP ointment in patients with cancer receiving chemotherapy, a systematic review will be performed.

\section{OBJECTIVES}

We aimed to propose a protocol for a systematic review to evaluate the effectiveness and safety of FLP ointment for the treatment of NSCLC.

\section{METHODS AND ANALYSIS \\ Criteria \\ Types of studies}

Any available randomised controlled trials (RCTs) in English and Chinese, both published and unpublished, will be included in the review.

\section{Types of participants}

Patients diagnosed with NSCLC for the first time will be included. The patients with stage III and IV tumours will be included, regardless of their age, sex, and ethnicity. NSCLC must be diagnosed based on the The National Comprehensive Cancer Network (NCGN) guideline by pathology and/or cytology methods.

\section{Types of interventions}

Studies reporting FLP ointment treatment will be included. The control group will consist of patients given no treatment, placebo ointment, or other conventional treatment including chemotherapy, radiotherapy, and targeted therapy. Trials that evaluate the effects of FLP ointment used in combination with another therapy will also be included in the review.

\section{Outcomes}

\section{Primary outcomes}

The primary outcomes will be the therapeutic effects of treatment according to standards for the treatment of solid tumours by the WHO and quality of life as evaluated by Karnofsky score and weight.

\section{Secondary outcomes}

Secondary outcomes will include: (a) syndrome according to standards for evaluating TCM; (b) safety based on 
adverse effects; (c) peripheral blood counts of immunocompetent cells, specifically activity of natural killer cells and the T lymphocyte subset and (d) survival time.

\section{Search methods for identification of studies Databases}

The electronic databases to be searched will include MEDLINE, the Cochrane Central Register of Controlled Trials (CENTRAL) in the Cochrane Library, the Excerpt Medica Database (EMBASE), the China National Knowledge Infrastructure (CNKI), China Scientific Journal Database (VIP), the Wanfang Database, and the Chinese Biomedical Literature Database (CBM) from inception to 2016 in English or Chinese.

\section{Searching other resources}

We will also search for other relevant systematic review articles and trials. The cited manuscripts will be manually searched once one systematic review is included.

\section{Data collection and analysis \\ Selection of studies}

The manuscripts will be included without restrictions on language or publication status from inception to 2016. The search terms consist of: non-small cell lung cancer, FLP ointment, chemotherapy, and RCTs (table 1).

\begin{tabular}{ll} 
Table 1 & Search strategy used in Pubmed database \\
\hline No & Search items \\
\hline 1 & Carcinoma, Non-Small Cell Lung \\
2 & Carcinoma, Non Small Cell Lung \\
3 & Carcinomas, Non-Small Cell Lung \\
4 & Lung Carcinoma, Non-Small Cell Lung \\
5 & Lung Carcinomas, Non-Small Cell Lung \\
6 & Non-Small Cell Lung Carcinomas \\
7 & Non-Small Cell Lung Cancer \\
8 & Non-Small Cell Lung Carcinoma \\
9 & Non-Small Cell Lung Carcinoma \\
10 & Carcinoma, Non-Small Cell Lung \\
11 & Non-Small Cell Lung Cancer \\
12 & 1 or 2-11 \\
13 & fei-liu-ping \\
14 & FLP ointment \\
15 & 13 or 14 \\
16 & Medicine, Chinese Traditional \\
17 & Traditional Chinese Medicine \\
18 & Chinese Medicine, Traditional \\
19 & Chung I Hsueh \\
20 & Hsueh, Chung I \\
21 & Zhong Yi Xue \\
22 & Chinese Traditional Medicine \\
23 & Traditional Medicine, Chinese \\
24 & 16 or 17-23 \\
25 & 12 and 15 or 24 \\
\hline
\end{tabular}

\section{Data extraction and management}

Two authors ( $\mathrm{HZ}$ and $\mathrm{SH}$ ) will be responsible for the data extraction independently following the criteria in the Cochrane Handbook for Systematic Reviews of Intervention (V.5.1.0).

Excluded studies and the reasons for exclusion will be listed in a table. Discrepancies during the process of selection will be resolved by discussion, and a consensus will be reached by a third person (RL). New information will be transferred into Review Manager 5.3.

\section{Assessment of risk of bias}

Two authors ( $\mathrm{HZ}$ and $\mathrm{SH}$ ) will independently assess the methodological quality of included trials. The methodological quality of the included RCTs will be assessed according to the guidance of the Cochrane Handbook for Systematic Review of Interventions (V.5.1.0), which includes the following criteria: selection bias caused by random sequence generation and allocation concealment, performance bias caused by blinding of participants and personnel, detection bias caused by blinding of outcome assessments, attrition bias caused by incomplete outcome data, and reporting bias caused by selective outcome reporting. Consensus will be reached via discussion with a third author (RL) in case of discrepancies. If necessary, we will contact the authors for missing data, methods of blinding, and randomisation.

\section{Measurements of treatment effect}

HRs will be used to summarise survival data. Risk ratios will be calculated for dichotomous data, and weighted mean differences will represent continuous outcomes. For both types of data, $95 \%$ CIs will be calculated.

\section{Addressing missing data}

If relevant data are missing, we will contact the authors to request the missing data. If those relevant data are not acquired, those data will be excluded from the analysis. Sensitivity analyses will be explored to evaluate how meta-analysis results change if these were excluded. In addition, we will discuss the influence of missing data on the findings of the review.

\section{Assessment of heterogeneity}

We will use $\chi^{2}$ test and $\mathrm{I}^{2}$ statistic test to assess the heterogeneity. If the $\mathrm{I}^{2}$ value is $<50 \%$ or $\mathrm{p}>0.10$, we will pool data using a fixed-effect model. Otherwise, a random effects model will be used, or we will conduct a descriptive analysis. If substantial heterogeneity is identified, this point will be listed and potential reasons will be assessed using subgroup analyses.

\section{Assessment of reporting bias}

If 10 or more studies are included in the meta-analysis, funnel plots will be used to detect reporting bias and poor methodological quality of small studies. The Egger method ${ }^{19}$ will be also used to explain the asymmetry. 


\section{Data synthesis}

Statistical analyses will be performed using RevMan manager 5.3 software (The Cochrane Collaboration). If there is no substantial statistical heterogeneity $\left(\mathrm{I}^{2}<50 \%\right)$, a fixed-effect model will be applied. Otherwise, we will use the random-effect model to reach a conclusion. If significant heterogeneity is detected, we will search for the clinical and methodological causes and provide an explanation. Otherwise, we will exclude the data and conduct a systematic narrative synthesis to summarise the findings of the included studies.

\section{Subgroup analysis}

If the data are available and sufficient, we will also conduct subgroup analyses. Relevant subgroups might include those treated with: FLP ointment versus placebo ointment or no treatment; FLP ointment versus conventional chemotherapy; FLP ointment plus conventional chemotherapy versus conventional chemotherapy only; FLP ointment versus chemoradiotherapy; FLP ointment plus chemoradiotherapy versus chemoradiotherapy; FLP ointment versus target therapy; and FLP ointment plus target therapy versus target therapy. Data will be compared between patients of different sexes and between stages (diagnosed with III or IV stage).

\section{Sensitivity analysis}

We also conduct sensitivity analysis to verify the study conclusions. The reliability of the conclusions will be determined according to the methodological qualities, the sample size, and the option of using missing data. ${ }^{20}$

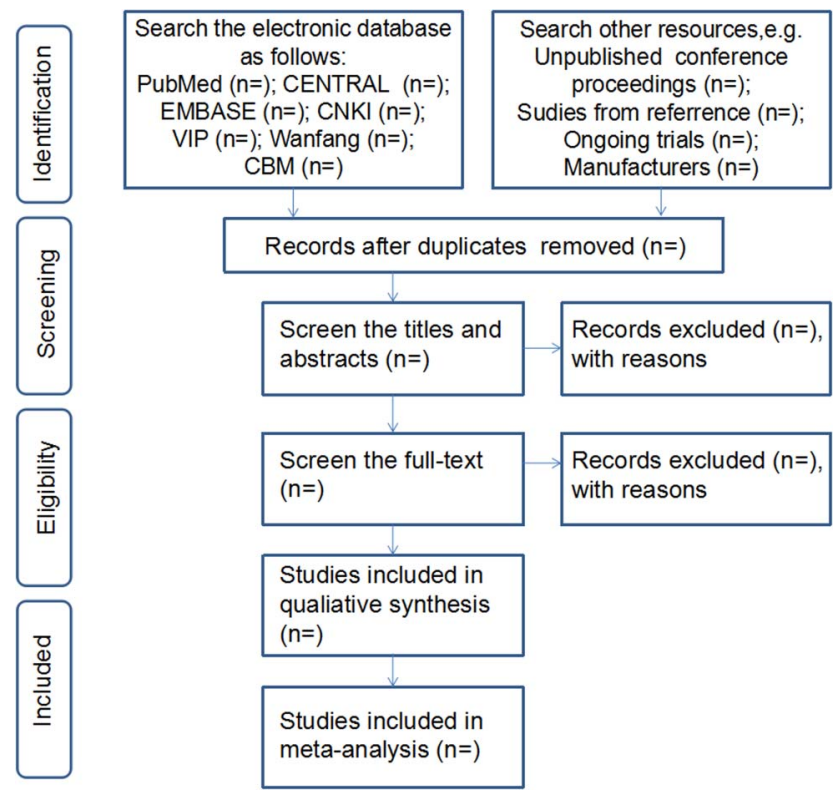

Figure 1 Process of the systematic review. CBM, Chinese Biomedical Literature Database; CENTRAL, Cochrane Central Register of Controlled Trials; CNKI, China National Knowledge Infrastructure; EMBASE, Excerpt Medica Database; VIP, China Scientific Journal Database.

\section{Ethics and dissemination}

Ethical approval is not required for the proposed meta-analysis, because the data used in this systematic review will not concern the privacy of individual patient. We will disseminate the results of this systematic review by publishing the manuscript in a peer-reviewed journal or presenting the findings at a relevant conference.

\section{DISCUSSION}

Chemotherapy causes significant adverse effects on patients' activities and quality of life, and FLP ointment is widely used in China to improve the relevant symptoms. However, there has been no systematic review of the efficacy of treatment with FLP ointment. Therefore, it is necessary to perform an objective systematic review to assess the efficacy and safety of FLP ointment in the treatment of NSCLC.

We provide a flow chart for this systematic review (figure 1). Our review may provide evidence for researchers and be helpful for clinical practitioners in treating NSCLC. The systematic review will also have obvious limitations, especially the language bias. Medical studies prepared in Japanese and Korean will not be covered in this review.

Contributors $\mathrm{HZ}$ and $\mathrm{SH}$ contributed to the conception of the study. $\mathrm{HZ}$ and SH wrote the draft of manuscript, and was revised by XX, TX, SC, QG, YG and $\mathrm{BH}$. The search strategy was developed by all of the authors, $\mathrm{HZ}$ and $\mathrm{SH}$ will search, extract data, assess the risk of bias, and complete the data synthesis. RL will arbitrate in case of disagreement and ensure the absence of errors. All authors approved the publication of the protocol.

Funding This work was supported by the National Natural Science Foundation of China (grant numbers 81273718 and 81102719).

Disclaimer The funders had no influence on the study design, data collection and analysis, as well as the right to publish.

Competing interests None declared.

Provenance and peer review Not commissioned; externally peer reviewed.

Data sharing statement The findings of this systematic review will be disseminated via peer-reviewed publications and conference presentations. Please contact the corresponding author for further information if the unpublished data from this study are available.

Open Access This is an Open Access article distributed in accordance with the Creative Commons Attribution Non Commercial (CC BY-NC 4.0) license, which permits others to distribute, remix, adapt, build upon this work noncommercially, and license their derivative works on different terms, provided the original work is properly cited and the use is non-commercial. See: http:// creativecommons.org/licenses/by-nc/4.0/

\section{REFERENCES}

1. American Cancer Society. Cancer facts and figures 2007. Atlanta: American Cancer Society, 2007.

2. Siegel RL, Miller KD, Jemal A, et al. Cancer statistics, 2016. CA Cancer J Clin 2016;66:7-30.

3. $\mathrm{Ni} \mathrm{YH}, \mathrm{Li} \mathrm{X}, \mathrm{Xu} \mathrm{Y}$, et al. Chinese herbal medicine for advanced pancreatic cancer. The Cochrane Library. John Wiley \& Sons, Ltd, 2012.

4. http://seer.cancer.gov/statfacts/html/lungb.html.

5. Datta $D$, Lahiri B. Preoperative evaluation of patients undergoing lung resection surgery. Chest 2003;123:2096-103. 
6. Nam KY, Choi JE, Hong SC, et al. Recent progress in research on anticancer activities of Ginsenoside-Rg3. Korean J Pharmacognosy 2014;45:1-10.

7. Howlader N, Noone A, Krapcho M, et al. SEER Cancer Statistics Review, 1975-2009 (Vintage 2009 Populations) based on November 2011 SEER data submission. Bethesda, MD: National Cancer Institute, 2012. http://seer.cancer.gov/csr/1975 2009 pops09/

8. Traditional medicine-growing needs and potential. WHO Policy Perspectives on Medicines. 2002;(2):1-6.

9. Claxton M. Culture, health and civilization. In: Leander B, ed. Culture and health. Paris: UNESCO Pub, 1996:17-46.

10. Auyeung KK, Han QB, Ko JK. Astragalus membranaceus: a review of its protection against inflammation and gastrointestinal cancers. Am J Chin Med 2016;44:1-22.

11. Poudyal D, Cui X, Le PM, et al. A key role of microRNA-29b for the suppression of colon cancer cell migration by American ginseng. PLOS ONE 2013;8:e75034.

12. Qian Y. Effects of removing stasis medicinal herbs on tumorigenesis and metastasis. J Tradit Chinese Med 2008;49:942-5.

13. Yang B, Wang YQ, Cheng RB, et al. Induction of cytotoxicity and apoptosis in human gastric cancer cell SGC-7901 by isovaltrate acetoxyhydrin isolated from Patrinia heterophylla Bunge involves a mitochondrial pathway and G2/M phase cell cycle arrest. Asian Pac $J$ Cancer Prev 2013;14:6481-6.

14. Zhang P, Piao B, Sun G. [Influence of feiliuping No. 2 on platelet surface glycoprotein expression in mid-late stage lung cancer patients]. Zhongguo Zhong Xi Yi Jie He Za Zhi 2000;20:31-3.
15. Zheng HG, Piao BK, Hua BJ. [Effect of feiliuping ointment and its disassembled prescriptions on dendritic cell of mice with transplanted Lewis lung cancer]. Zhongguo Zhong Xi Yi Jie He Za Zhi 2010;30:1288-91.

16. Li W, Chen C, Saud SM, et al. Fei-Liu-Ping ointment inhibits lung cancer growth and invasion by suppressing tumor inflammatory microenvironment. BMC Complement Altern Med 2014:14:919-25.

17. Rui L, Zheng H, Li W, et al. Anti-tumor enhancement of Fei-Liu-Ping ointment in combination with celecoxib via cyclooxygenase-2-mediated lung metastatic inflammatory microenvironment in Lewis lung carcinoma xenograft mouse model. J Transl Med 2015;13:1-14.

18. Mountzios G, Aravantinos G, Alexopoulou Z, et al. Lessons from the past: long-term safety and survival outcomes of a prematurely terminated randomized controlled trial on prophylactic vs. hemoglobin-based administration of erythropoiesis-stimulating agents in patients with chemotherapy-induced anemia. Mol Clin Oncol 2016;4:211-20.

19. Deeks JJ, Higgins JPT, Altman DG. Analysing data and undertaking meta-analysis. In: Higgins JPT, Green S, eds. Cochrane Handbook for Systematic Reviews of Interventions Version 5.1.0 [updated March 2011]. The Cochrane Collaboration, 2011. http://www cochrane-handbook.org (accessed Jun 2014).

20. Egger M, Davey Smith G, Schneider M, et al. Bias in meta-analysis detected by a simple, graphical test. BMJ 1997;315:629-4. 